CERN-TH/97-253

hep-ph/9709489

\title{
Power counting in exclusive quarkonium decays
}

\author{
Gerhard A. Schuler ${ }^{a}$ \\ Theory Division, CERN, CH-1211 Geneva 23, Switzerland
}

\begin{abstract}
Recent progress in the factorization of exclusive decays of heavy quarkonia into long-distance and short-distance contributions is presented. The role of higher Fock states and colour-octet contributions is outlined.
\end{abstract}

To appear in the Proceedings of the High-Energy Physics Euroconference on Quantum Chromodynamics Montpellier, France, 3-9 July 1997

${ }^{a}$ Heisenberg Fellow.

CERN-TH/97-253

September 1997 



\title{
Power counting in exclusive quarkonium decays
}

\author{
G.A. Schuler ${ }^{\text {a* }}$ \\ aTheory Division, CERN, CH-1211 Geneva 23, Switzerland
}

Recent progress in the factorization of exclusive decays of heavy quarkonia into long-distance and short-distance contributions is presented. The role of higher Fock states and colour-octet contributions is outlined.

\section{INTRODUCTION}

Exclusive reactions at large momentum transfer $Q$ can be calculated in perturbative QCD (pQCD) owing to a factorization theorem [1] that separates the short-distance physics of the partonic subreactions at the scale $Q$ from the longerdistance physics associated with the binding of the partons inside the hadrons. The full amplitude is given as a sum of terms, where each term factors into two parts, a hard-scattering amplitude $T_{H}$, calculable in pQCD, and wave functions $\psi\left(x_{i}, \mathbf{k}_{\perp \mathbf{i}}\right)$ for each hadron $H$ :

$\mathcal{M}=\sum_{n} T^{\mathrm{H}} \times \prod_{i} \psi_{i}$

The separate dependence on the factorization scale $\mu_{F}$ cancels, to the order considered, in the full amplitude $\mathcal{M}$.

The importance of the various terms in (1.1) depends on their scaling with $1 / Q$. For the contribution that has the weakest fall-off with $Q$ (leading-twist contribution), the amplitude $T_{H}$ describes the scattering of clusters of collinear partons from the hadron and is given by valenceparton scatterings only. Hence the only nonperturbative input required are the distribution amplitudes (DAs) $\phi_{H}\left(x_{i}, Q\right)$ for finding valence quarks in the hadron, each carrying some fraction $x_{i}$ of the hadron's momentum. The DAs represent wave functions integrated over transverse momentum $\mathbf{k}_{\perp}$ up to a factorization scale $\mu_{F}$, of order $Q$, and obey the BL evolution equations [1]. Indeed, most calculations so far have been performed in this standard hard-scattering approach (sHSA) summarized by:

\footnotetext{
*Heisenberg Fellow.
}

- leading (i.e. valence) Fock states only;

- collinear approximation;

- (mostly) lowest-order in $\alpha_{s}(Q)$;

- tailored for $S$-wave hadrons (orbital angular momentum $L=0$ ). Up to now results of the sHSA remained at a qualitative level, lacking a rigorous discussion of (in particular the powerlike $\propto 1 / Q$ ) corrections. Power corrections can be classified as follows: (i) Corrections arising from the overlap of the soft wave functions; (ii) corrections associated with the transverse momentum of the partons inside the hadrons; (iii) corrections from higher Fock states.

Recently, exclusive processes at large scales have attracted new interest:

- New, precise data on the pion-photon transition form factor allow a quantitative extraction of the (non-perturbative) pion DA [2].

- A modified hard-scattering approach (mHSA) has been developed, which allows for the incorporation of transverse degrees of freedom and gluonic radiative corrections in the form of a Sudakov factor [3]. This does not only allow for a dynamical setting of the renormalization, $\alpha_{s}\left(\mu_{R i}\right)$, and factorization, $\mathrm{DA}_{i}\left(\mu_{F i}\right)$, scales. Now also the perturbative contribution to exclusive observables can be calculated self-consistently, i.e. without major contributions from phase-space regions, where the virtualities of the internal partons become soft and perturbation theory is not applicable.

- A new factorization approach for inclusive quarkonium decays (and production) has been developed [4. Colour-octet contributions associated with higher Fock components are found to 

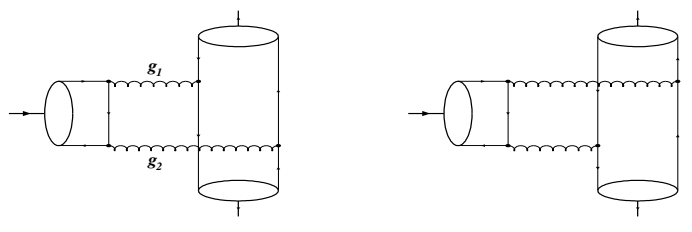

Figure 1. Feynman graphs for the colour-singlet decay $\chi_{\mathrm{c} J} \rightarrow \pi \pi(J=0,2)$.

be crucial.

- The importance of higher Fock states also for exclusive quarkonium decays has been realized and new power-counting rules derived [5].

\section{2. $\chi_{\mathrm{c} J} \rightarrow \pi \pi$ in the mHSA}

The decay $\chi_{\mathrm{c} J}$ into a pair of pseudoscalar mesons is given by the Feynman diagrams in Fig. 1, describing the annihilation of the dominant Fock state of $\chi_{\mathrm{c} J}$ into a pair of gluons. For $J^{P C}=J^{++}$mesons, the valence Fock state is a ${ }^{3} P_{J}$ colour-singlet $c \overline{\mathrm{c}}$ pair (in the spectroscopic notation ${ }^{2 S+1} L_{J}$ of a spin angular-momentum state). Decays of heavy quarkonia can be calculated in the HSA since the heavy-quark mass $m_{\mathrm{c}}$ provides the hard scale $Q=m_{\mathrm{c}}$. The sHSA result has been known for a long time [6]

$\Gamma\left[\chi_{\mathrm{c} J} \rightarrow \pi \pi\right]=c_{J} m_{\mathrm{c}} \alpha_{s}^{4}\left(\frac{f_{\pi}}{m_{\mathrm{c}}}\right)^{4} \frac{\left|R_{P}^{\prime}(0)\right|^{2}}{m_{\mathrm{c}}^{5}}$,

where $c_{J}$ results from a convolution of the pion DAs with the hard kernel of Fig. 田, $f_{\pi}$ is the pion decay constant, and $R_{P}^{\prime}(0)$ the derivative of the coordinate wave function at the origin.

Recently, the calculations of the decay widths in the mHSA have been completed [5]. The colour-singlet decay amplitude within the mHSA is calculated from the amplitude

$$
\begin{array}{r}
M^{(1)}\left(\chi_{\mathrm{c} J} \rightarrow \pi \pi\right) \sim\left(\frac{\delta_{i j}}{\sqrt{3}} \int \mathrm{d}^{3} k \tilde{\Psi}_{J}^{(1)} S_{J}^{(1)}\right) \\
\int \mathrm{d} x \mathrm{~d} y \int \mathrm{d}^{2} \mathbf{b}_{1} \mathrm{~d}^{2} \mathbf{b}_{2} \exp [-S] \\
\hat{\Psi}_{\pi}^{*}\left(y, \mathbf{b}_{2}\right) \hat{T}_{H J}\left(x, y, \mathbf{b}_{1}, \mathbf{b}_{2}\right) \hat{\Psi}_{\pi}\left(x, \mathbf{b}_{1}\right) .
\end{array}
$$

This is the convolution w.r.t. the momentum fractions $x, y$ and transverse separation scales $\mathbf{b}_{1}, \mathbf{b}_{2}$ of the two pions; $\hat{T}_{H J}$ is the Fourier transform of the hard-scattering amplitude $T_{H J}$ with the transverse $\left(k_{\perp}\right)$ dependence retained, see Fig. 1. The Sudakov factor $\exp [-S]$ takes into account those gluonic radiative corrections not accounted for in the QCD evolution of the wave function. Finally, the $\chi_{\mathrm{c} J}$ are treated non-relativistically: the bracket in (2.3) contains the colour-singlet and spin projection operators, and $\tilde{\Psi}_{J}^{(1)}$ are the (reduced) non-relativistic wave functions, which yield $R_{P}^{\prime}(0)$ after integration over the relative c $\overline{\mathrm{c}}$ momentum $k$. The factorization and renormalization scales depend on the virtualities of the respective adjacent fermion propagators, e.g.

$\mu_{R i}^{2}=\max \left\{4(1-x)(1-y) m_{\mathrm{c}}^{2}, \frac{1}{b_{1}^{2}}, \frac{1}{b_{2}^{2}}\right\}$.

The results of the improvement of the mHSA calculation over the sHSA one can be summarized as follows: first, (soft) end-point contributions are severely suppressed. Indeed, the results are self-consistent in the sense that only about $2 \%$ of the decay rate originate from phase-space regions where $\alpha_{s}\left(\mu_{R 1}\right) \alpha_{s}\left(\mu_{R 2}\right)>1 / 2$. Secondly, the uncertainty of the result against variation of the input parameters of the calculation (shape parameter $B_{2}$ of the pion-wave function parameter, charm quark mass, and QCD scale $\Lambda_{\mathrm{QCD}}$ ) is reduced in the mHSA compared to the sHSA but still sizeable, see Table 1. However, even with an optimistic parameter choice the theoretical prediction falls short by a factor of 4 , calling for an additional contribution.

\section{Inclusive decays of heavy quarkonia}

The non-relativistic quark-potential model is very successful in describing the static properties of heavy quarkonia. A quarkonium $\left|H\left(J^{P C}\right)\right\rangle$ is considered to be pure, obviously coloursinglet, $\left|Q \bar{Q}\left({ }^{2 S+1} L_{J}\right)\right\rangle$ state where the $Q \bar{Q}$-pair is bound by an instantaneous potential. The nonrelativistic nature of the bound system implies the existence of three important scales (besides the heavy-quark mass $m$ ): (the modulus of) the heavy-quark three momentum $\sim m v$, the quark's kinetic energy $\sim m v^{2}$, and the QCD scale $\Lambda_{\mathrm{QCD}}$. Inclusive quarkonium decays into light hadrons 
Table 1

Decay widths of $\chi_{\mathrm{c} J} \rightarrow \pi^{+} \pi^{-}$for various choices of the parameters compared with data.

\begin{tabular}{|c|c|c|c|c|}
\hline$B_{2}$ & $m_{\mathrm{c}}$ & $\Lambda_{\mathrm{QCD}}$ & $\overline{\Gamma\left(\chi_{\mathrm{c} J} \rightarrow \pi^{+}\right.}$ & $\left.\pi^{-}\right)[\mathrm{keV}]$ \\
\hline & [GeV] & [GeV] & $J=0$ & $J=2$ \\
\hline \multicolumn{5}{|c|}{ Modified HSA } \\
\hline 0 & 1.5 & 0.2 & 8.22 & 0.41 \\
\hline 0.1 & 1.5 & 0.2 & 12.13 & 0.53 \\
\hline-0.1 & 1.5 & 0.2 & 5.61 & 0.33 \\
\hline 0 & 1.8 & 0.2 & 2.54 & 0.12 \\
\hline 0 & 1.35 & 0.2 & 15.3 & 0.78 \\
\hline 0 & 1.5 & 0.15 & 4.34 & 0.21 \\
\hline 0 & 1.5 & 0.25 & 13.1 & 0.68 \\
\hline \multicolumn{5}{|c|}{ Experiment } \\
\hline & PDG|7 & & $105 \pm 30$ & $3.8 \pm 2.0$ \\
\hline & BES $\sqrt[8]{8}$ & & $62.3 \pm 17.3$ & $3.04 \pm 0.73$ \\
\hline
\end{tabular}

are accessible to perturbative QCD: for sufficiently large $m$, the annihilation time $\sim 1 / m$ is much smaller than the time scales relevant to $Q \bar{Q}$ binding. Hence the short-distance annihilation of the $Q \bar{Q}$ pair into gluons and light quarks can be separated from the long-distance effects of $Q \bar{Q}$ binding. The basis of this separation is provided by an effective field theory, NRQCD, supplemented by perturbative factorization [1]. The inclusive decay rate is given as a double expansion in $\alpha_{s}(m)$ and $v^{2}$, to leading order in $\Lambda_{\mathrm{QCD}} / m$.

The factorization makes use of the fact that there are, in any Feynman diagram, some propagators that are off shell by an amount $\sim m^{2}$ much larger than the scales governing $Q \bar{Q}$ binding. Hence, in the effective low-energy theory these may be contracted to a point. The remainder of the diagram involves only distances much larger than $1 / m$. For the case of inclusive decays, these are related to expectation values of universal long-distance matrix elements (MEs) $\mathcal{O}_{n}$. The $\mathcal{O}_{n}$ are well-defined 4 -fermion operators of NRQCD of the form $\mathcal{O}_{n}=\left(\psi^{\dagger} K_{n} \chi\right)\left(\chi^{\dagger} K_{n}^{\prime} \psi\right)$. The inclusive decay widths thus take on the form

$\Gamma=\sum_{n} f_{n}\left\langle H\left|\mathcal{O}_{n}\right| H\right\rangle$,

where the short-distance coefficients $f_{n}$ are calculable as perturbation series in $\alpha_{s}(m)$.
The importance of the various long-distance MEs in (3.5) is given by their scaling with the heavy-quark velocity $v$. The velocity-scaling (power-counting) rules were first established based on consistency requirements of the NRQCD field equations [9]. More rigorously, these follow from a multipole expansion in the wavelength of the emitted (multiple) gluon field $\lambda=R_{H} / \lambda_{g}$. In NRQCD, $\lambda \sim k_{g} /(m v) \sim v$. This formulation has the advantage that it can be generalized to the situation where $\Lambda_{\mathrm{QCD}}$ is not much smaller than $m v^{2}$ (as assumed in NRQCD) giving rise to different scaling rules 10].

The $\mathrm{J} / \psi$ is dominantly an $S$-wave state. Its Fock-state expansion starts as

$$
\begin{aligned}
\left|\mathrm{J} / \psi\left(1^{--}\right)\right\rangle & =O(1)\left|c \overline{\mathrm{c}}_{1}\left({ }^{3} S_{1}\right)\right\rangle \\
+ & O(v)\left|c \overline{\mathrm{c}}_{8}\left({ }^{3} P_{J}\right) \mathrm{g}\right\rangle+O\left(v^{2}\right),
\end{aligned}
$$

where the subscripts 1 and 8 denote colour-singlet and colour-octet states, respectively. Annihilation into light hadrons via the valence Fock state involves the $\mathrm{ME}\left\langle\mathrm{J} / \psi\left|\mathcal{O}_{1}\left({ }^{3} S_{1}\right)\right| \mathrm{J} / \psi\right\rangle$, which we take to scale as $v^{0}=1$ (dividing out an overall $v^{3}$ scaling). In the potential model this corresponds to $\left|R_{S}(0)\right|^{2}$. The first corrections for $S$-wave quarkonia arise from higher-dimensional operators (suppressed by $v^{2}$ ) still involving the leading Fock component. These lead, for example, to differences between the $\mathrm{J} / \psi$ and $\eta_{\mathrm{c}}$ wave functions. The colour-octet component of (3.6) enters first at relative order $v^{4}$. Nonetheless, its contribution can be important, namely when the short-distance coefficients of the singlet states are suppressed by powers of $\alpha_{s}(m)$ and/or kinematical factors. An example is $\mathrm{J} / \psi$ production at high transverse momentum [11].

The situation is different for $\chi_{\mathrm{c} J}$, as becomes evident from their Fock-state expansion

$$
\begin{aligned}
\left|\chi_{\mathrm{c} J}\left(J^{++}\right)\right\rangle & =O(1)\left|c \overline{\mathrm{c}}_{1}\left({ }^{3} P_{J}\right)\right\rangle \\
+ & O(v)\left|\mathrm{c} \overline{\mathrm{c}}_{8}\left({ }^{3} S_{1}\right) \mathrm{g}\right\rangle+O\left(v^{2}\right) .
\end{aligned}
$$

The $v^{2}$ factor of the $E 1$ multipole suppression of the $c \bar{c} g$ state is compensated by the fact that a $P$ wave operator scales as $v^{2}$ relative to an $S$-wave operator. Hence, contrary to the case of $S$-wave decays, two 4 -fermion operators contribute to the decay rate of $P$-wave states into light hadrons at 


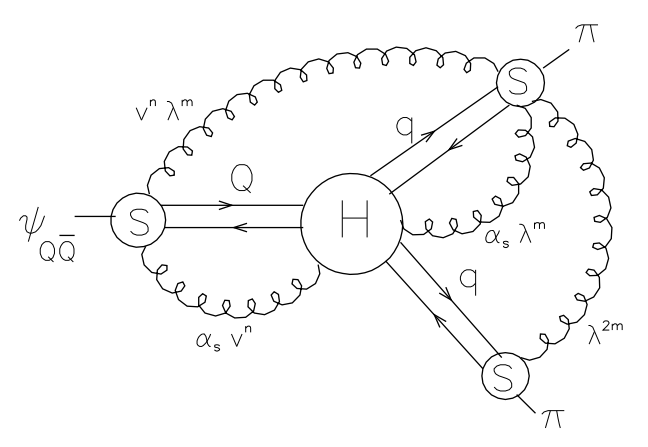

Figure 2. Factorization of $\chi_{\mathrm{c} J} \rightarrow \pi \pi$.

leading order in $v$

$$
\begin{aligned}
\Gamma\left[\chi_{\mathrm{c} J} \rightarrow \mathrm{LH}\right]=\frac{c_{1}}{m_{\mathrm{c}}^{4}}\left\langle\chi_{\mathrm{c} J}\left|\mathcal{O}_{1}\left({ }^{3} P_{J}\right)\right| \chi_{\mathrm{c} J}\right\rangle \\
+\frac{c_{8}}{m_{\mathrm{c}}^{2}}\left\langle\chi_{\mathrm{c} J}\left|\mathcal{O}_{8}\left({ }^{3} S_{1}\right)\right| \chi_{\mathrm{c} J}\right\rangle+O\left(v^{2} \Gamma\right) .
\end{aligned}
$$

The term involving the colour-singlet matrix element is the one familiar from the quark-potential model. Indeed, up to corrections of order $v^{2}$, one has $\left\langle\chi_{\mathrm{c} J}\left|\mathcal{O}_{1}\left({ }^{3} P_{J}\right)\right| \chi_{\mathrm{c} J}\right\rangle=9\left|R_{P}^{\prime}(0)\right|^{2} /(2 \pi)$.

The decays of $P$-wave (and higher orbital-angular-momentum states) probe components of the quarkonium wave function that involve dynamical gluons. However, the contribution to the inclusive annihilation rate from the higher Fock state $|c \bar{c} g\rangle$ is parametrized through a single number, namely the expectation value of the octet operator between the $\chi_{\mathrm{c} J}$ state, i.e. the colour-octet matrix element in (3.8).

\section{Factorization of exclusive quarkonium decays}

In contrast to inclusive decays, there are now two possibilities to absorb long-distance contributions, into either quarkonium-specific or lighthadron-specific objects. In order to set up a factorization scheme for exclusive quarkonium decays one has to consider the virtualities occurring in an arbitrary Feynman diagram. Three regions have to be distinguished. Propagators can be off
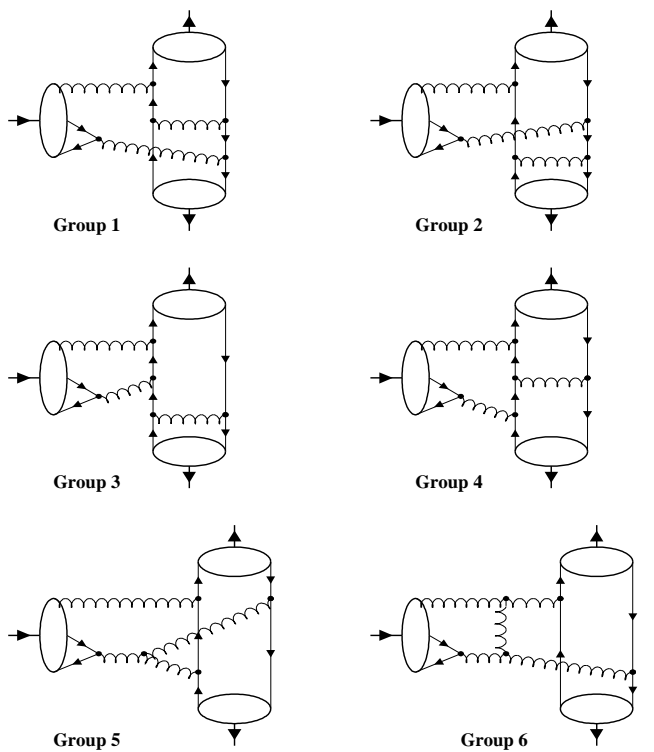

Figure 3. Some colour-octet contributions to $\chi_{\mathrm{c} J} \rightarrow \pi \pi$.

shell by order $m^{2},(m v)^{2}$, or $\Lambda_{\mathrm{QCD}}^{2} \equiv(\lambda m)^{2}$. Correspondingly, such contributions have to be associated with the hard amplitude, the quarkonium, or the light hadrons, respectively. This then leads to a triple expansion in $\alpha_{s}(m), v$, and $\lambda$.

Another difference with inclusive hadronic decays is the fact that the factorization into shortdistance and long-distance contributions holds at the amplitude level rather than at the level of decay rates (i.e. squared amplitudes). In this sense factorization of exclusive decays resembles more the case of inclusive electromagnetic decays, where the final-state particles are also colour singlets.

Higher Fock components in light hadrons certainly exist. Consider the decay $\chi_{\mathrm{c} J} \rightarrow \pi \pi$, Fig. 3. The (multipole-based) Fock-state expansion of the pion starts as follows

$$
\begin{aligned}
& \left|\pi\left(0^{-+}\right)\right\rangle=O(1)\left|\mathrm{q} \overline{\mathrm{q}}_{1}\left({ }^{1} S_{0}\right)\right\rangle+O(\lambda)\left|\mathrm{q} \overline{\mathrm{q}}_{8}\left({ }^{1} P_{1}\right) \mathrm{g}\right\rangle \\
& \quad+O\left(\lambda^{2}\right)\left\{\left|\mathrm{q} \overline{\mathrm{q}}_{8}\left({ }^{3} S_{1}\right) \mathrm{g}\right\rangle+\ldots\right\}+O\left(\lambda^{3}\right) .
\end{aligned}
$$


Since both octet terms give corrections to the leading one of the same order $\lambda^{2}$, two new (nonperturbative) light-cone DAs had to be specified in a calculation using higher Fock components of the pion. Clearly, not much predictive power can be expected. In fact, higher Fock contributions to the pion $2^{2}$ are power-suppressed $\left(\propto \Lambda_{\mathrm{QCD}}^{2} / m^{2}\right)$ and hence become negligible for sufficiently large quark masses.

In contrast, higher Fock components of heavy quarkonia are suppressed by powers of $v$ only, or even scale as the leading Fock contribution. Moreover, owing to the non-relativistic expansion one does not need new DAs but only new 'decay constants' (expectation values of NRQCD operators). Of course, there remains the problem of colour conservation. A perturbative factorization can be set up for the case $\alpha_{s}(\mu) / \pi \ll 1$ for $\mu \sim m v$ since this allows one to treat the constituent gluon(s) as the constituent (valence) quarks. Colour conservation is then achieved by coupling the constituent gluon (in all possible ways) to the hard process, see Fig. 3 .

The calculation of $\chi_{\mathrm{c} J} \rightarrow \pi \pi$ in the collinear approximation leads, however, to singular results. This is not surprising as the Feynman diagrams of Fig. 3 must contain contributions, which, to leading order in $\alpha_{s}$, constitute the two higher Fock states $q \bar{q} g$ of the pion. The regularization of the propagators associated with the 'would-be' constituent gluon of the pion depends on the hierarchy of $\lambda$ and $v^{2}$ : the singularities are protected by either relativistic correction $\propto m v^{2}$ to the heavy-quark momentum or the finite transverse momentum $k_{\perp} \propto \Lambda_{\mathrm{QCD}}=\lambda m$ of the (light) valence quarks of the pion.

In 15 $v^{2} \ll \lambda(\ll v)$ was assumed, in which case it is the non-zero value of $k_{\perp}$ taken into account in the mHSA that acts as a regulator and, in turn, determines the size of the colour-octet contribution to the decay width. The only new non-perturbative parameter is then a single number, namely the octet decay constant $f^{(8)}$ enter-

$\overline{2 \text { The situation may be }}$ different for decays into higher- $L$ mesons.
Table 2

Results for the $\chi_{\mathrm{c} J}$ decay widths (in $\mathrm{keV}$ ) into pions $\left(f^{(8)}=1.46 \times 10^{-3} \mathrm{GeV}^{2} ; B_{2}=0\right)$ in comparison with experimental data.

\begin{tabular}{lccc}
\hline & & PDG [7] & BES [3] \\
\hline$\chi_{\mathrm{c} 0} \rightarrow \pi^{+} \pi^{-}$ & 45.4 & $105 \pm 47$ & $64 \pm 21$ \\
$\chi_{\mathrm{c} 2} \rightarrow \pi^{+} \pi^{-}$ & 3.64 & $3.8 \pm 2.0$ & $3.04 \pm 0.73$ \\
$\chi_{\mathrm{c} 0} \rightarrow \pi^{0} \pi^{0}$ & 23.5 & $43 \pm 18$ & \\
$\chi_{\mathrm{c} 2} \rightarrow \pi^{0} \pi^{0}$ & 1.93 & $2.2 \pm 0.6$ & \\
\hline
\end{tabular}

Table 3

Results for the $\chi_{\mathrm{c} J}$ decay widths into K's and $\eta$ 's in comparison with experimental data $\left(f^{(8)}=\right.$ $\left.1.46 \times 10^{-3} \mathrm{GeV}^{2}, B_{1}^{\mathrm{K}}=0\right)$.

\begin{tabular}{|c|c|c|}
\hline & $J=0$ & $=2$ \\
\hline & \multicolumn{2}{|c|}{$\bar{\Gamma}\left[\chi_{\mathrm{c} J} \rightarrow \mathrm{K}^{+} \mathrm{K}^{-}\right] \quad[\mathrm{keV}]$} \\
\hline$B_{2}^{\mathrm{K}}=-0.176$ & 22.4 & 1.68 \\
\hline$B_{2}^{\overline{\mathrm{K}}}=-0.100$ & 38.6 & 2.89 \\
\hline PDG $|7|$ & $99 \pm 49$ & $3.0 \pm 2.2$ \\
\hline BES [8] & $52 \pm 17$ & $1.04 \pm 0.43$ \\
\hline & \multicolumn{2}{|c|}{$\Gamma\left[\chi_{\mathrm{c} J} \rightarrow \eta \eta\right] \quad[\mathrm{keV}]$} \\
\hline$B_{2}^{\eta}=-0.036$ & 24.0 & 1.91 \\
\hline$B_{2}^{\eta}=0.000$ & 32.7 & 2.66 \\
\hline PDG [7] & $35 \pm 20$ & $1.6 \pm 1.0$ \\
\hline
\end{tabular}

ing the $|c \bar{c} g\rangle$ wave function

$\left|\chi_{\mathrm{c} J}^{(8)}\right\rangle=\frac{t_{i j}^{a}}{2} f^{(8)} \int \mathrm{d} z_{1} \mathrm{~d} z_{2} \Phi_{J}^{(8)}\left(z_{1}, z_{2}, z_{3}\right) S_{J \nu}^{(8)}(4$

where $t=\lambda / 2$ is the Gell-Mann colour matrix and $a$ the colour of the gluon. With this single parameter $\chi_{\mathrm{c} J}$ decays into all pairs of pseudoscalar mesons can be predicted. The $f^{(8)}$ value obtained by fitting to the four decays into pions (Table 2) corresponds to a very reasonable probability of the octet $|c \bar{c} g\rangle$ state, $P_{\text {c } \bar{c} g} \sim 0.5$. Predictions for $\chi_{\mathrm{c} J}$ decays into K's and $\eta$ 's also agree nicely with data, see Table 3. Predictions for $\Upsilon$ decays have been obtained as well (Table (i).

It should be emphasized that the colour-octet contribution from the $|c \bar{c} g\rangle$ Fock state is numerically important, in accordance with the fact that 
Table 4

Decay widths in eV for $P$-wave bottomonia for two values of $f^{(8)} \quad\left(m_{b}=4.5 \mathrm{GeV} ; R_{P}^{\prime}(0)=\right.$ $\left.0.7 \mathrm{GeV}^{5 / 2}\right)$.

\begin{tabular}{lcc}
\hline$f^{(8)}\left[\mathrm{GeV}^{2}\right]$ & $\chi_{\mathrm{b} 0} \rightarrow \pi^{+} \pi^{-}$ & $\chi_{\mathrm{b} 2} \rightarrow \pi^{+} \pi^{-}$ \\
\hline $1.46 \times 10^{-3}$ & 20.6 & 1.69 \\
$5 \times 10^{-3}$ & 152 & 13.8 \\
\hline$f^{(8)}\left[\mathrm{GeV}^{2}\right]$ & $\chi_{\mathrm{b} 0} \rightarrow \pi^{0} \pi^{0}$ & $\chi_{\mathrm{b} 2} \rightarrow \pi^{0} \pi^{0}$ \\
\hline $1.46 \times 10^{-3}$ & 10.5 & 0.88 \\
$5 \times 10^{-3}$ & 78.2 & 7.27 \\
\hline
\end{tabular}

it is not suppressed relative to the contribution from the valence Fock state, either in $\Lambda_{\mathrm{QCD}} / m$ or in $v$.

\section{Summary}

It is now possible to obtain accurate predictions for exclusive reactions involving pseudoscalar mesons. On the one hand, the nonperturbative input, their DAs, can well be determined from recent precise measurements of the meson-photon transition form factors. On the other hand, with mHSA an improved framework exists for calculations of the contributions of the leading Fock state. Hence, the leading coloursinglet contributions are well under control.

In the case of the charmonium $\chi_{\mathrm{c} J}$ decays into a pair of pseudoscalars, one finds that the coloursinglet contribution alone is not sufficient to accommodate the data. Indeed, it turns out that the colour-octet contribution from the $|c \bar{c} g\rangle$ Fock state contributes at the same level as the coloursinglet one. Its inclusion yields good agreement with experimental data.

In general, one can expect colour-octet contributions to be important if the contribution from the leading Fock state is suppressed. This happens for quarkonia with orbital-angular momentum $L \geq 1$. It occurs also if decays of the leading Fock state into massless quarks $\left(m_{q}=0\right)$ are forbidden by a symmetry (such as $G$-parity or helicity) so that these seemingly leading decays are actually power-suppressed $\propto\left(m_{q} / m_{Q}\right)^{n}$.

The study [5] is a first attempt to derive a fac- torization approach for exclusive decays of heavy quarkonia. More work is needed to generalize the factorization to higher orders in $\alpha_{s}\left(m_{Q}\right), v$, and $\Lambda_{\mathrm{QCD}} / m$. In particular it has to be shown that the various contributions in an arbitrary Feynman diagram can be disentangled in a systematic way.

Acknowledgements:

I am pleased to thank J. Bolz and P. Kroll for an enjoyable collaboration.

\section{REFERENCES}

1. S.J. Brodsky and G.P. Lepage, Phys. Rev. D22 (1980) 2157.

2. R. Jakob, P. Kroll and M. Raulfs, J. Phys. G22 (1996) 45;

S. Ong, Phys. Rev. D52 (1995) 3111;

I.V. Musatov and A.V. Radyushkin, JLABTHY-97-07, February 1997, hep-ph/9702443; S.J. Brodsky et al., SLAC-PUB-7473, May 1997, hep-ph/9705221.

3. J. Botts and G. Sterman, Nucl. Phys. B325 (1989) 62;

H.-N. Li and G. Sterman, Nucl. Phys. B381 (1992) 129.

4. G.T. Bodwin, E. Braaten and G.P. Lepage, Phys. Rev. D51 (1995) 1125.

5. J. Bolz, P. Kroll and G.A. Schuler, Phys. Lett. B392 (1997) 198; preprint CERN-TH/97-64, April 1997, hep-ph/9704378.

6. A. Duncan and A.H. Mueller, Phys. Lett. B93 (1980) 119.

7. Particle Data Group: Review of Particle Properties, Phys. Rev. D54 (1996) 1.

8. Y. Zhu for the BES coll., talk given at the XXVIII Int. Conf. on High Energy Physics, 25-31 July 1996, Warsaw, Poland.

9. G.P. Lepage et al., Phys. Rev. D46 (1992) 4052.

10. G.A. Schuler, preprint CERN-TH/97-12, January 1997, hep-ph/9702230;

M. Beneke, preprint CERN-TH/97-55, March 1997, hep-ph/9703429.

11. E. Braaten, S. Fleming and T.C. Yuan, preprint OHSTPY-HEP-T-96-001, February 1996, hep-ph/9602374. 